\title{
Functional Decline as an Indicator of III-Health: A Retrospective Case Study of the Process Leading to Lung Cancer
}

\section{Lesleigh Kowalski}

Department of Family Medicine, University of Washington, Seattle, WA, 98195, USA
Correspondence: Lesleigh Kowalski Frank Email lkowalsk@uw.edu
Purpose: Lung cancer claims more lives than any cancer in the world and remains difficult to diagnosis at early stages. Detecting lung cancer is challenging due to nonspecific symptom presentation. Literature was reviewed to consider functional decline as an indicator for illhealth. This study explored the process experienced from recognition in a change of health to receiving a lung cancer diagnosis from a patient's perspective in order to examine this phase through a biopsychosocial lens.

Patients and Methods: A single-case design methodology was used for this study. The method of data collection was semi-structured interviews with people diagnosed with lung cancer utilizing criterion sampling. The case study was bound by diagnostic and geographical factors to frame the single-case: participants were limited to those living in Alaska diagnosed with stage III or stage IV lung cancer.

Results: One $(n=1)$ person participated in this study. Themes consistent with lung cancer detection process from a patient's perspective include symptom denial, symptom reductionism, and gradual impact on function.

Conclusion: Although the number of participants was extremely limited due to the COVID19 pandemic at the time of recruitment, this case study suggests a decline in function present prior to being diagnosed with lung cancer. Opportunities exist within the provider and patient interface to promote earlier detection include educating medical providers to ask specific, closed-ended, non-disease related functional questions to ascertain more details and a holistic representation of patients' health. Raising public awareness of lung cancer symptoms, such as fatigue and dyspnea, is also warranted.

Keywords: lung cancer, early diagnosis, primary health care

\section{Introduction}

Lung cancer claims more lives than any cancer in the world. ${ }^{1}$ Detecting lung cancer is challenging because patients typically visit their healthcare provider with nonspecific symptoms, exhibiting signs such as weight loss, dyspnea, fatigue, or cough. ${ }^{2,3}$ The vagueness of these symptoms and the many comorbidities that may exist make providing an accurate diagnosis difficult, which leads to many missed diagnoses and patient delays. Ninety-percent of people diagnosed with lung cancer will die of the disease, yet early stage lung cancer can be treated surgically with increased survival rates. ${ }^{4}$ However, only $15 \%$ of lung cancers are diagnosed at stage I, making surgical resection unlikely. ${ }^{5,6}$ Late-stage lung cancer diagnoses are even more prevalent in minority communities. ${ }^{7}$ 
The underlying pathological processes that leads to symptoms of lung cancer likely also causes changes in functional participation in activities of daily living, mental health, and effect on roles at work and home. Weight loss, as seen through cancer cachexia, is associated with functional impairment and decreased survival rates after diagnosis. ${ }^{8}$ Skeletal metastases are often present in lung cancer as well, especially in more advanced stages, which may cause pain and an inability to perform certain tasks, therefore creating potential for diagnostic discussion to ascertain the possibility of cancer. ${ }^{9}$ At the time of diagnosis, patients with bone metastases exhibit an increase in pathological fractures and a decreased survival rate. ${ }^{10}$ However, minimal research focuses on these functional factors prior to lung cancer diagnoses as potential indicators of illness.

Previous research utilized directed interviews of 22 patients diagnosed with lung cancer to map their prediagnosis history as a way to test the hypothesis that the route to diagnosis differed between those with operable versus non-operable lung cancer. ${ }^{11}$ The study found systemic symptoms were experienced by people with operable and non-operable disease, yet lack of awareness did not necessarily cause a help-seeking response from health changes that impacted participation in activities of daily living. ${ }^{11}$ To understand these findings more fully, a followup study specifically examined the psychosocial factors leading to a delay in seeking treatment by those later diagnosed with lung cancer. ${ }^{5}$ In the follow-up qualitative study, a change in functional participation went unnoticed by providers due to patients not reporting them from lack of awareness; the symptoms were typically attributed to "everyday causes" such as smoking and not ill-health, and a general sense of unworthiness due to a positive smoking history. ${ }^{5}$

Few other studies exist in which the methodology includes interviewing patients diagnosed with cancer in order to establish a tangible process of retrospective studies from the patients' perspective of their pre-diagnostic process. Lung cancer is underrepresented within that small group of studies, which typically focus on breast cancer. ${ }^{12-14}$ Nevertheless, studies of delay in breast cancer diagnosis provide relevant and useful information. For example, it is largely understood in public health perceptions that a lump found in the breast requires immediate medical attention, ${ }^{13}$ yet similar awareness of single alarm symptom equivalent to this for lung cancer does not exist. Additionally, this research sheds light on women suppressing their own symptoms in order to care for their family, thereby prolonging their inevitable diagnosis. ${ }^{13}$ Given that the rate of lung cancer is rising in women, this finding is an especially important pattern to remember because women are at a higher risk for developing lung cancer when compared to their smoking and non-smoking male counterparts. ${ }^{15}$ Also, lung cancer among women has yet to reach its peak given the historical context of smoking uptake. ${ }^{16}$

Although no retrospective studies prior to diagnosis from a functional perspective exist, many studies examine function throughout people's cancer journey after diagnosis. For example, a meta-analysis found 107 studies examining pre/rehabilitation on patients with NSCLC with focus on physical fitness, which indicates rehabilitation for the lung cancer population is widely studied. ${ }^{17}$ Only eleven studies fit the criteria of this particular metaanalysis, yet the quantity of current research remains high indicating function throughout cancer treatment is a heavily researched area of health. For example, research assessed physical activity throughout the first six months of those diagnosed with non-small cell lung cancer. ${ }^{18}$ Results indicate that at diagnosis individuals with lung cancer were weaker, more depressed, and less active than their non-diagnosed counterparts. ${ }^{18}$ These symptoms may therefore be evident prior to diagnosis. Another study recommends prehabilitation prior to cancer surgery as a shift away from the paradigm of rest before surgery, with focus on exercise, mental health, and optimizing nutrition, which found prehabilitation enhances postsurgical recovery. ${ }^{19}$ The progression of this research demonstrates a backwards approach: first through rehabilitative methods after or during treatment, then prehabilitative after diagnosis and before treatment, and now it is time to examine the pre-diagnosis period.

One may wonder why pre-diagnostic research is necessary with low-dose computed tomography (LDCT) lung cancer screening as an option; however, the United States Preventive Services Task Force (USPSTF) LDCT lung cancer screening recommendation does not stand alone and is intended to be implemented as part of a greater program of care. ${ }^{4}$ Additionally, the USPSTF LDCT lung cancer screening is only recommended for some patients based on age and smoking history, and is not a comprehensive solution. Furthermore, the uptake of LDCT lung cancer screening has been extremely low, with less than $5 \%$ of the eligible population actually receiving LDCT lung cancer screening. ${ }^{20}$ The screening 
rate is lower in areas that lack access to care, such as Alaska, ${ }^{21}$ the geographical setting of this study. To provide the necessary knowledge to diagnosing providers, both technological and nontechnological strategies are needed with a focus on basic clinical skills, such as data gathering and synthesizing the information. ${ }^{22}$ Research reports that primary care physicians may not feel knowledgeable about how to ask questions regarding certain functional measures and that patients' answers are unreliable as the patient may not recognize if a decline is present. ${ }^{23} \mathrm{Or}$, the patient may not even report the symptoms. ${ }^{23}$ Some present symptoms to providers without knowing the symptoms represent illness ${ }^{24}$ therefore, it is vital that providers can attach meaning to these symptoms. Yet many delays in lung cancer diagnosis are due to perceptions of the symptoms by diagnosing providers. ${ }^{25}$ The misperception is not necessarily a lack of knowledge but a lack of phrasing or an undervaluation of the importance that functional participation in meaningful activities plays in the role of health and illness.

The purpose of this study is to utilize the patient's perspective to describe the process a person undergoes from recognition of an initial change in health to receipt of a lung cancer diagnosis, define characteristics of participants' functional presentation prior to receiving a diagnosis of lung cancer, and identify biopsychosocial factors that contribute to a delayed lung cancer diagnosis. This case study aims to establish feasibility for future research. The terms late-stage and delayed diagnosis of lung cancer are defined as those diagnosed at stage III or IV lung cancer regardless of type. This study explores and describes the process rather than determining causality.

\section{Theoretical Framing}

The Andersen Model of Total Patient Delay is a guiding model for considering the research aims and analyzing the subsequent data. This is a frequent model cited to frame patient related influences on diagnostic delay, which breaks up the patient-caused delay into five stages: appraisal delay, illness delay, behavioral delay, scheduling delay, and treatment delay. $^{26}$ This is based on the Psychophysiological Comparison Theory, which assumes people are motivated to maintain a psychophysiological equilibrium and will seek explanation for unexplained signs and symptoms. ${ }^{26} \mathrm{~A}$ recent systematic review analyzed studies applying the Andersen Model of Total Patient Delay, and recommended modifications to the stages into appraisal, help-seeking, diagnostic, and pre- treatment with continuous processes occurring throughout the delay. ${ }^{24}$ The Modified Andersen Model of Total Patient Delay is seen as a descriptive, rather than predictive, model of observing delays in the patient/provider continuum. The Modified Andersen Model of Total Patient Delay is then used to inform the context of patient/provider delays rather than predict outcomes. An important takeaway from the Modified Andersen Model of Total Patient Delay is the emphasis on the non-linear path many cancer diagnoses may take. ${ }^{24}$

The Modified Andersen Model of Total Patient Delay is applied to the diagnostic journey in the study below to explore lengths of time in each interval. Through this lens, it is possible to assess delays that occurred and investigate the causes within each interval. The Modified Andersen Model of Total Patient Delay is used to inform the analysis and direct future research. Certain interview questions such as, "What prompted you to seek help?" directly tie to the transition from one phase (appraisal) to the next (helpseeking) and illuminate the underlying processes. The Modified Andersen Model of Total Patient Delay applies to all types of cancer, yet only one study included in the systematic review involved lung cancer, and this was combined with breast, brain, gastrointestinal, gynecological, head, and neck cancers, therefore more research is needed specifically assessing the unique aspects of lung cancer. ${ }^{24}$ Utilizing this model may facilitate additional knowledge for the purpose of lung cancer and diagnostic intervals.

\section{Patients and Methods}

The researcher applied a single-case design methodology for this study. The method of data collection was semistructured interviews with people diagnosed with lung cancer utilizing criterion sampling. The case study was bound by diagnostic and geographical factors to frame the single-case: participants were limited to those living in Alaska diagnosed with stage III or stage IV lung cancer. This particular population was chosen in order to examine the process of late-stage lung cancer diagnoses. Procedures followed were in accordance with the ethical standards of relevant institutional or national bodies. The research protocol was approved by the appropriate ethical committees. Written informed consent was provided by the participant to have the case details and recorded responses published.

\section{Recruitment}

Recruitment began in August 2019 after approval from institutional review boards. Recruitment ceased in 
March 2020 due to the COVID-19 pandemic and the need to protect vulnerable populations from community transmission.

\section{Participants}

A total of one person participated in this study. The participant was a 63-year-old Caucasian male diagnosed with Stage IIIB non-small cell adenocarcinoma lung cancer in February 2019. The participant smoked one pack of cigarettes per day for 12 years, from the ages of 21-33. He quit smoking 30 years ago. Comorbidities include high cholesterol, hormonal imbalance, sleep apnea, and prior history of skin cancer. He works as a Licensed Clinical Social Worker (LCSW) and holds a Master's degree in Social Work and a Master's degree in Human Resource Management. He was married (17 years) without children. Family history includes cancer, alcoholism, and COPD.

\section{Procedures}

The semi-structured interview occurred on November 12, 2019 at the participant's place of employment in his office and spanned two hours. The recorded interview incorporated both semi-structured and structured questions (Appendix A). The researcher administered the Mini$\operatorname{Cog} \odot$ Screening for Cognitive Impairment in Older Adults to assess his ability to recall. The participant scored a $3 / 3$, demonstrating adequate recall as a reliable historian. NVivo transcription software transcribed the interview followed by manual transcription by the researcher to account for errors. The original recording was then deleted.

The researcher manually analyzed the transcription and coded for primary themes. The analysis included inductive coding, in which the responses were coded manually lineby-line and then separated into categories working from the ground up. ${ }^{27}$ The categories reflect the overarching themes to represent the process prior to diagnosis. The qualitative data was analyzed through open coding procedures typically used in grounded theory research. ${ }^{28}$

\section{Results}

Three themes emerged that describe the process the participant experienced from recognition of an initial change in health to receipt of a lung cancer diagnosis, that occurred over a period of two years:

- Denial of symptoms

- Symptom reductionism
- Gradual impact in function

\section{Denial}

The participant mentioned his own denial on six different instances throughout the interview when asked what prompted him to seek help and how he explained his symptoms to his PCP. On other mentions, the participant voluntarily brought up being in denial regarding his fear of having COPD.

I was in big denial about being short of breath .... I mean, again, the shortness of breath thing, that is all about denial .... my wife insists that I have had shortness of breath for a couple of years prior to my diagnosis .... Again, I was so sensitive towards COPD that I just kept going, I was in a big denial about being short of breath.

When asked to provide his best guesstimate on how long he felt it went on for, he replied, "probably for two, three years. Okay maybe even longer, three or four years."

\section{Symptom Reductionism}

The participant experienced different symptoms that he interpreted as being separate, not interconnected, leading to symptom reductionism. He began to feel "tired," "run down," and "flu-like" prior to January 2019. He said he "just didn't look well." He attributed feeling ill to working long hours.

The participant showed pictures on his iPhone taken prior to his diagnosis.

It's only in hindsight since diagnosis that those pictures kind of make sense, but I just seemed overly, like maybe a little thin, not standing up real tall, just kind of depleted in some way.

He exhibited a dry, non-productive cough. He scheduled an appointment with his primary care provider (PCP) prior to leaving on a vacation in January 2019 . He reports his PCP prescribed a Zithromax Z-pak and told him "to have fun [on vacation] and get some sun."

While on his vacation, the participant remembers experiencing chest/back pain. He assumed he "pulled a muscle on my shoulder blade" and did not consider the pain as unusual, or significant even when considering his other symptoms as a whole.

Then in the shower I coughed and spit out a bit of blood, which I thought was interesting .... but even with the blood I never thought about the possibility of lung cancer. 
After returning from vacation, the participant went to his PCP again.

I figured I'd give the good doctor a call as soon as I get back on the mainland. We flew into Seattle the next day on the way to Anchorage, and I called his office. He said, 'Yeah come on in when you get back to Anchorage,' and I did. He said, 'Well I really think it's a blood clot. Everything you're telling me, and your family history, and that you just got off a plane, I think it is a travelrelated blood clot.'

The participant went to the emergency room to receive imaging for the blood clot, and it was there that his lung cancer was discovered. "It was actually one of the last things on my mind because, again, I did what they had told me to do 30 years earlier which was to quit smoking," he said.

\section{Gradual Impact on Function}

The participant described three notable changes in function prior to diagnosis. He did not report difficulty with his basic activities of daily, such as dressing and hygiene, yet his sleep was impacted.

Sleeping was rather difficult. I kept coughing trying to open that up to breath. I had a wedge pillow .... if I slept on my left side I was okay because [in hindsight] the gravity would pull the tumor over and it would open that up so I could breathe without coughing.

Second, he experienced a decrease in activity tolerance. When asked if he needed to cut back on social engagements or work, he replied: "I felt like I needed to include more exercise. Aerobics exercise specifically." Two years prior to his diagnosis, the participant trained for a bear viewing and fishing trip that took place two years before he received the diagnosis. Then, he stopped exercising and gradually began taking the elevator instead of the stairs. He began to feel "winded on long walks" with his wife and their dog, which were cut progressively shorter to accommodate his increasing fatigue.

Third, his tolerance at work decreased. This functional change is the cue to action that brought him into see his PCP, as he was not able to keep up with his typical work demands. In doing so, he felt he had, "a bug, the flu, whatever." Prior to his activity tolerance at work decreasing, his decreased activity tolerance that impacted the duration of his walks with his wife and preference for the elevator rather than stairs did not cause an alarm, despite seeing his PCP throughout this time period. The participant did not mention these facts to his PCP, and his PCP did not ask.

The participant denies any mental health or cognitive changes or peripheral neuropathy prior to his diagnosis. However, he reports an impactful and memorable moment while on vacation prior to his diagnosis.

I don't know why, because I've been around helicopters my whole life. I've been up in helicopters before that particular time, and maybe it was just that we were flying we were down into a lot of canyons and high up into the mountains. We were flying in canyons and it was above us, I just thought 'this is not a good place to be if there's a problem, right.' But I was definitely white knuckling it. I think I just had a premonition, I just had a sense of my own mortality in that situation. I don't know, and few days later I was told I had cancer.

Denial, reductionism of symptoms, and a gradual impact on function describe the process the participant experienced from recognition of a change in health to receiving a lung cancer diagnosis.

\section{Discussion}

Although the number of participants is extremely limited, this study suggests the method and methodology may be useful to answer the research questions. When reviewing the process the participant experienced from recognition of an initial change in health to receipt of his lung cancer diagnosis, it is evident that the process unfolded over approximately two years. The process was gradual, and incorporated a slow progression of a decrease in activity tolerance until acute flu-like symptoms prompted him to seek help. The symptoms were considered isolated events, and not considered interconnected, typical in reductionistic medicine in which individual parts are assessed separately rather than the whole. ${ }^{29}$ Previous research similarly found "disconnected interpretations of bodily changes" (p. 1384) in regards to simultaneous symptoms assumed unrelated by participants. ${ }^{11}$ It is important to connect these symptoms as numerous synchronous symptoms are common among people with lung cancer. ${ }^{2,30}$

Per his report, the participant attended appointments regularly during the two years prior to his lung cancer diagnosis to manage other health concerns. Whether or not missed opportunities ${ }^{31}$ existed is unknown without review of his medical records. What is clear, though, is 
the lack of communication between the participant and his PCP regarding his health as a whole, as he neither provided the information about his decline of activity tolerance, sleeping positional difficulties (although he was diagnosed with sleep apnea), and dyspnea, and, per the participant's report, his PCP did not ask. The lack of communication between the provider and patient reflects research supporting the training of non-disease specific, closed-ended questions from PCPs to patients to ascertain increased symptom information. ${ }^{32}$ This finding also reflects research that indicates patients are less likely to report changes in health considered "lifestyle" and PCPs are less likely to specifically ask. ${ }^{10,23}$ As the participant said, "I just didn't talk about that stuff." An important opportunity exists in that by educating healthcare providers to inquire deeper into the function of an individual, they may uncover additional clues that promote earlier diagnosis of disease. Additionally, educating patients to increase awareness of their functional changes presents another opportunity for care improvement and the potential for meaningful conversations with their healthcare providers.

In the participant's case, a known familial history of lung cancer did not increase the participant's health and help seeking behavior related to his dyspnea, yet he practiced health-seeking behavior for other health issues. The disconnect appears to reside in his perceived susceptibility for lung cancer, for which he did not perceive he was at risk due to prior smoking cessation. He said, "I am not trying to make up excuses, but I am not even eligible for the screening." Therefore the need may exist to expand screening guidelines to include more former smokers. Another disconnect occurs in his perceived barriers, in which fear of a COPD diagnosis outweighed the benefits of bringing up his dyspnea and fatigue to his PCP. After the process, he exhibits self-efficacy of a cancer survivor as he said twice in the interview that he is, "not going anywhere yet."

When comparing the participant's process to The Modified Andersen Model of Total Patient Delay, the delay in this case resides in the Appraisal interval as he detected bodily changes, contemplated his bodily changes, and considered discussing these with his PCP as a sign of illness. ${ }^{24}$ As represented in the Model, the interval was fluid and cyclical. Once he perceived a reason to discuss his bodily changes with his PCP, he was diagnosed within 30 days (given time for his vacation, then the 10-day wait for appropriate biopsy to be done as the surgeon was on vacation during that time). Diagnostic and Pre-Treatment factors did not lead to a delay in diagnosis, per the participant's report of the timeline of events. From presentation to his PCP and receipt of diagnosis, the total diagnostic period was less than 30 days, which is within recommendations from countries that provide diagnostic timeline guidelines. ${ }^{33}$

In review of the participant's process, certain biopsychosocial factors contributed to his delayed lung cancer diagnosis. The term delayed is used here as he recognized symptoms 90 days prior to seeking to help. Biologically, the participant may be genetically predisposed to developing cancer given his family history. ${ }^{34}$ His father died of leukemia and his eldest brother died of lung cancer. He smoked for 12 years, which increased his risk for developing lung cancer. From a psychosocial perspective, denial and fear contributed to his delayed diagnosis, which are common causes of delayed diagnoses. ${ }^{24,35}$ Fear often leads to either a delay or early help-seeking behaviors. ${ }^{35,}{ }^{36} \mathrm{He}$ was aware of his risk for COPD, and feared receiving that diagnosis. The fear stems from witnessing his brother fight COPD, who later received a bilateral lung transplant and succumbed to lung cancer 7-8 months after the lung transplant in 2006 due to an unknown tumor in his lymph node that spread to his new lungs. This experience made him, "more concerned about getting COPD like my oldest brother."

The participant's social support system indicated awareness of his decreasing health. The participant is married and his wife repeatedly mentioned his dyspnea.

She saw me kind of come up winded on long walks that we would take ... and I think, you know, she had concerns about my being short of breath. Again, I don't think it was about cancer at that point I think she thought it COPD.

When asked if she encouraged him to seek medical help for the issue, he said, "No, she knows how strong my denial is and how stubborn I am." Despite her lack of urging to seek medical care, her acknowledgement of his symptoms promoted his awareness. This seemed to neither promote nor prevent his diagnostic interval. However, a systemic review of marital status and stage of cancer found people who are married are more likely to have cancer diagnosed at an earlier stage than their unmarried counterparts, ${ }^{37}$ therefore the participants' wife may have promoted a positive effect in his diagnostic interval. 


\section{Conclusion}

The participant experienced a process that unfolded over a two-year period prior to receiving a lung cancer diagnosis. His process included a gradual progression of dyspnea, and with that a gradual decrease in activity tolerance that manifested itself through less active choices (ie, elevator over stairs, lower threshold at work) as well as changes in sleep. The symptoms that prompted him to seek care were fatigue and feeling generally ill with a dry cough. Once he presented himself to his PCP, his lung cancer diagnosis was made in a timely manner and treatment began within 30 days of his diagnosis. Opportunities within the provider and patient interface to promote earlier detection include educating PCPs to ask specific, closed-ended, non-disease related functional questions to ascertain more details and a holistic representation of patients' health. Raising public awareness of lung cancer symptoms, such as fatigue and dyspnea, is also warranted.

\section{Limitations}

This study is limited by the number of participants. However, the participant serves as an exemplar of people living the experience and the information obtained from the participant's interview is important in understanding the process of functional decline prior to receiving a lung cancer diagnosis. Including all stages of lung cancer in future research may be helpful to compare processes between late-stage and early-stage diagnoses as well as facilitate a larger participant size.

Also, this interview took place nine months after his diagnosis, which poses limitations to recall accuracy. Recall bias may impact the meaning of signs, symptoms, or experiences in retrospective studies, ${ }^{38}$ and therefore exists as a limitation as well to the participants' retrospective perception of the process he experienced. Additionally, as another limitation to providing holistic data, the participant did not feel comfortable providing his pre-diagnostic medical records to include in this study.

\section{Implications}

This study indicates the need to research the process experienced prior to lung cancer diagnosis further with a larger participant cohort to establish results with increased reliability. This study also suggests people experience symptoms for several years prior to presenting with a cue to action, and implies lung cancer is not a silent disease. They may experience several symptoms at once that appear unrelated. Similar to other findings, results denote ill-health is not considered until there is a breaking point that forces help-seeking behavior. ${ }^{10,11}$ This demonstrates the need for increased awareness of lung cancer progression and lung cancer symptoms among the general population and healthcare providers. Recent studies similarly discussed the need for campaigns that specifically target symptom awareness and education in patients who are considered high risk for lung cancer, rather than widespread national campaigns. ${ }^{21,39}$ Additionally, the lack of communication regarding the participant's nondisease specific symptoms imply the need to create a simple functional assessment for use in primary care, with emphasis on functional participation in activities of daily living and objective measurements of dyspnea and fatigue. This may be created utilizing research to inform a questionnaire or checklist, which has shown to promote a higher standard of baseline performance in healthcare. ${ }^{40}$ This may facilitate communication between patients and PCPs to uncover symptoms that patients typically fail to mention on their own. Future research should focus on this as well.

\section{Acknowledgments}

The author would like to acknowledge and thank the participant in this work for their time and willingness to share their story. The author conducted this study during her doctoral dissertation and acknowledges Drs. Jennifer Antick, Nancy Krusen, Aaron Kusano, and Matthew Thompson for their guidance.

\section{Disclosure}

The author reports no conflicts of interest in this work.

\section{References}

1. Cancer Facts and Figures; 2019. Available from: https://www.cancer. org/content/dam/cancer-org/research/cancer-facts-and-statistics /annual-cancer-facts-and-figures/2019/cancer-facts-and-figures-2019. pdf. Accessed April 24, 2020.

2. Hamilton W, Peters TJ, Round A, Sharp D. What are the clinical features of lung cancer before the diagno+sis is made? A population-based case-control study. Thorax. 2005;60(12):1059. doi:10.1136/thx.2005.045880

3. Lyratzopoulos G, Wardle J, Rubin G. Rethinking diagnostic delay in cancer: how difficult is the diagnosis? Br Med J. 2014;349(349): g7400. doi:10.1136/bmj.g7400

4. Moyer VA. Screening for lung cancer: u.S. preventive services task force recommendation statement. Ann Intern Med. 2014;160(5):330. doi:10.7326/M132771

5. Corner J, Hopkinson J, Roffe L. Experience of health changes and reasons for delay in seeking care: a UK study of the months prior to the diagnosis of lung cancer. Soc Sci Med. 2005;62(6):1381-1391. doi:10.1016/j.socscimed.2005.08.012 
6. Cifu AS, Davis AM. Lung cancer screening. $J$ Am Med Assoc. 2014;312(12):1248. doi:10.1001/jama.2014.12272

7. Bergamo C, Lin JJ, Smith C, et al. Evaluating beliefs associated with late-stage lung cancer presentation in minorities. J Thorac Oncol. 2013;8:12-18. doi:10.1097/JTO.0b013e3182762ce4

8. Lau S, Gannavarapu BS, Carter K, et al. Impact of socioeconomic status on pretreatment of weight loss and survival in non-small-cell lung cancer. J Oncol Pract. 2018;14(4):e211-e220. doi:10.1200/ JOP.2017.025239

9. Tsuya A, Kurata T, Tamura K, Fukuoka M. Skeletal metastases in non-small cell lung cancer: a retrospective study. Lung Cancer. 2007;57(2):229-232. doi:10.1016/j.lungcan.2007.03.013

10. Oliveira M, Marques B, Matos R, Fontenelle C, Mello F, Paschoal M. Pathological fractures due to bone metastases from lung cancer: risk factors and survival. Acta Ortop Bras. 2018;26(6):388-393. doi:10.1590/1413-785220182606201669

11. Corner J, Hopkinson J, Fitzsimmons D, Barclay S, Muers M. Is late diagnosis of lung cancer inevitable? Interview study of patients' recollections of symptoms before diagnosis. Thorax. 2005;60 (4):314-319. doi:10.1136/thx.2004.029264

12. Bish A, Ramirez A, Burgess C, Hunter M. Understanding why women delay in seeking help for breast cancer symptoms. J Psychosom Res. 2005;58(4):321-326. doi:10.1016/j.jpsychores. 2004.10.007

13. Burgess CC, Hunter MS, Ramirez AJ. A qualitative study of delay among women reporting symptoms of breast cancer. Br J Gen Pract. 2001;51(473):967-971.

14. Unger-Saldaña $\mathrm{K}$, Infante-Castañeda $\mathrm{CB}$. Breast cancer delay: a grounded model of help-seeking behaviour. Soc Sci Med. 2011;72 (7):1096-1104. doi:10.1016/j.socscimed.2011.01.022

15. Siegfried JM. Women and lung cancer: does oestrogen play a role? Lancet Oncol. 2001;2(8):506-513. doi:10.1016/S1470-2045(01) 00457-0

16. Mukherjee S. The Emperor of All Maladies: A Biography of Cancer. New York, USA: Scribner; 2010.

17. Driessen E, Peeters M, Bongers B, et al. Effects of prehabilitation and rehabilitation including a home-based component on physical fitness, adherence, treatment tolerance, and recovery in patients with non-small cell lung cancer: a systematic review. Crit Rev Oncol Hematol. 2017;114:63-76. doi:10.1016/j.critrevonc.2017.03.031

18. Granger CL, McDonald CF, Irving L, et al. Low physical activity levels and functional decline in individuals with lung cancer. Lung Cancer. 2014;83(2):292-299. doi:10.1016/j.lungcan.2013.11.014

19. Carli F, Gillis C, Scheede-Bergdahl C. Promoting a culture of prehabilitation for the surgical cancer patient. Acta Oncol. 2017;56 (2):128. doi:10.1080/0284186X.2016.1266081

20. Jemal A, Fedewa SA. Lung cancer screening with low-dose computed tomography in the United States-2010 to 2015. JAMA Oncol. 2017;3(9):1278-1281. doi:10.1001/jamaoncol.2016.6416

21. Eberth JM, Bozorgi P, Lebrón LM, et al. Geographic availability of low-dose computed tomography for lung cancer screening in the United States, 2017. Prev Chronic Dis. 2018;15:E119. doi:10.5888/ pcd15.180241

22. Singh H, Giardina TD, Meyer A, et al. Types and origins of diagnostic errors in primary care settings. JAMA Intern Med. 2013;173 (6):418-425. doi:10.1001/jamainternmed.2013.2777

23. Wagland R, Brindle L, James E, Moore M, Esqueda AI, Corner J. Facilitating early diagnosis of lung cancer amongst primary care patients: the views of GPs. Eur J Cancer Care. 2017;26(3):e12704. doi:10.1111/ecc.12704
24. Walter FM, Webster A, Scott S, Emery J. The Anderson model of total patient delay: a systematic review of its application in cancer diagnosis. $J$ Health Serv Res Policy. 2012;17(2):110-118. doi:10.1258/jhsrp.2011.010113

25. Singh H, Sethi S, Raber M, Peterson L. Errors in cancer diagnosis: current understanding and future directions. J Clin Oncol. 2007;25 (31):5009-5018. doi:10.1200/JCO.2007.13.2142

26. Andersen BL, Cacioppo JT, Roberts DC. Delay in seeking a cancer diagnosis: delay stages and psychophysiological comparison processes. Br J Soc Psychol. 1995;34(1):33-52. doi:10.1111/j.20448309.1995.tb01047.x

27. Yin RK. Case Study Research and Applications: Design and Methods. 6th ed. California: SAGE; 2018.

28. Corbin J, Strauss A. Basics of Qualitative Research: Techniques and Procedures for Developing Grounded Theory. 3rd ed. California: SAGE; 2008.

29. Ahn A, Tewari M, Poon CS, Phillips RS. The limits of reductionism in medicine: could systems biology offer an alternative? PLoS Med. 2006;3(6):e208. doi:10.1371/journal.pmed.0030208

30. Walter FM, Rubin G, Bankhead C, et al. Symptoms and other factors associated with time to diagnosis and stage of lung cancer: a prospective cohort study. $B r J$ Cancer. 2015;112(S1):S6-13. doi:10.1038/bjc.2015.30

31. Singh H, Hirani K, Kadiyala H, et al. Characteristics and predictors of missed opportunities in lung cancer diagnosis: an electronic health record-based study. J Clin Oncol. 2010;28(20):3307-3315. doi:10.1200/JCO.2009.25.6636

32. Brindle L, Pope C, Corner J, Leydon G, Banerjee A. Eliciting symptoms interpreted as normal by patients with early stage lung cancer: could GP elicitation of normalized symptoms reduce delay in diagnosis? Cross-sectional interview study. BMJ Open. 2012;2: e001977. doi:10.1136/bmjopen-2012-001977

33. Kim JO, Davis F, Butts C, Winget M. Waiting time intervals for non-small cell lung cancer diagnosis and treatment in Alberta: quantification of intervals and identification of risk factors associated with delays. Clin Oncol ( $R$ Coll Radiol). 2016;28(12):750-759. doi:10.1016/j.clon.2016.06.010

34. El-Zein RA, Young RP, Hopkins RJ, Etzel CJ. Genetic predisposition to chronic obstructive pulmonary disease and/or lung cancer: important considerations when evaluating risk. Cancer Prev Res (Phila). 2012;5(4):522-527. doi:10.1158/1940-6207.CAPR-12-0042

35. Burgess CC, Ramirez AJ, Richards MA, Love SB. Who and what influences delayed presentation in breast cancer? Br J Cancer Supple. 1998;77(8):1343-1348. doi:10.1038/bjc.1998.224

36. de Nooijer J, Lechner L, de Vries H. A qualitative study on detecting cancer symptoms and seeking medical help; an application of Andersen's model of total patient delay. Patient Educ Couns. 2001;42:145-157. doi:10.1016/S0738-3991(00)00104-X

37. Buja A, Lago L, Lago S, Vinelli A, Zanardo C, Baldo V. Marital status and stage of cancer at diagnosis: a systematic review. Eur J Cancer Care. 2017;27:1-16.

38. Coughlin S. Recall bias in epidemiologic studies. J Clin Epidemiol. 1990;43:87-91. doi:10.1016/0895-4356(90)90060-3

39. Wagland R, Brindle L, Ewings S, et al. Promoting help-seeking in response to symptoms amongst primary care patients at high risk of lung cancer: a mixed method study. PLoS One. 2016;11(11): e0165677. doi:10.1371/journal.pone. 0165677

40. Gawande A. The Checklist Manifesto: How to Get Things Right. New York: Picador; 2010. 


\section{Publish your work in this journal}

The Journal of Multidisciplinary Healthcare is an international, peerreviewed open-access journal that aims to represent and publish research in healthcare areas delivered by practitioners of different disciplines. This includes studies and reviews conducted by multidisciplinary teams as well as research which evaluates the results or conduct of such teams or healthcare processes in general. The journal

covers a very wide range of areas and welcomes submissions from practitioners at all levels, from all over the world. The manuscript management system is completely online and includes a very quick and fair peer-review system. Visit http://www.dovepress.com/testimonials. php to read real quotes from published authors. 